\author{
ARTIFICIAL SATELLITES, Vol. 48. No. 4 - 2013 \\ DOI: 10.2478/arsa-2013-0014
}

\title{
SYMMETRIC NEUTRAL-ATMOSPHERE MAPPING FUNCTIONS: A REVIEW OF THE STATE-OF-THE-ART
}

\author{
M.A. Sharifi, A.H. Souri \\ (sharifi@ut.ac.ir, souri_rs@ut.ac.ir,) \\ Department of Surveying and Geomatics Engineering, University College of Engineering, \\ University of Tehran, North Kargar Ave., P.O. Box 11365-4563, Tehran, Iran
}

\begin{abstract}
.
The aim of this paper is to review of six recent symmetric mapping functions. The mapping function can be largely used for GPS meteorological measurements, InSAR atmospheric corrections and precise measurements of very long baseline interferometry (VLBI). These spacebased techniques use radio signal that propagate through the Earth's atmosphere. The electrically-neutral region, predominantly the troposphere, affects the speed and direction of travel of radio waves leading to existence of excess path. The mapping function models the elevation angle dependence of the delay. Within the past decade, significant improvements have been achieved in order to use of Numerical Weather Models (NWM) for geodetic positioning. Ray-tracing algorithms have been performed through refractivity shells retrieved from NWMs in order to relate zenith delays to slant delays. Therefore, there seems to be a real need for deep review of recent developments in the mapping function domain. This paper proposes a comprehensive review of the symmetric mapping functions state of the art, their spatio-temporal variations and used NWM and generic models. Niell Mapping Function (NMF), Vienna Mapping Function (VMF1), University of New Brunswick-VMF1 (UNB-VMF1) mapping functions, Global Mapping Function (GMF) and Global Pressure and Temperature (GPT2)/GMF are reviewed in this paper.
\end{abstract}

Keywords: Mapping functions, Numerical Weather Model, Geodetic measurements, Neutral atmosphere

\section{INTRODUCTION}

Space geodetic technologies such as Global Navigation Satellite System (GNSS), Very Long Baseline Interferometry (VLBI) and InSAR technique are widely used tool for making very precise geodetic measurements and mapping numerous geophysical phenomena on Earth. Particularly Global Positioning System (GPS) stations offer to provide total water column at high quality under all weather conditions with high temporal resolution which has been well-known as GPS meteorology (Emardson et al., 1998; Niell et al., 2001). These space-based techniques use radio signal that propagate through the Earth's atmosphere. The electrically-neutral region, predominantly the troposphere, affects the speed and direction of travel of radio waves. The neutral-atmosphere propagation delay is directly linked to the refractive index. The refractive 
index of a parcel of moist air is unlike from unity because its constituents experience polarization induced by the electromagnetic field of the radio signals (Mendez, 1999). The neutralatmosphere consists of dry gasses and water vapor. Dry gases vary smoothly both spatially and temporally. On the contrary, water is highly variable across the Earth's atmosphere. It is primarily found in the troposphere, which is the lowest 10-12 kilometers of the atmosphere. Nearly one half of total water vapor in atmosphere is found between sea level and $1.5 \mathrm{~km}$. less than $5-6 \%$ of water vapor is found above $5 \mathrm{~km}$ and less than $1 \%$ is found in the stratosphere $(>12 \mathrm{~km})$ (AGU, 1995). Water vapor has a permanent dipole moment that significantly contributes to the change of the refractive index. Although the dry constituents have no permanent dipole moment, their molecules are displaced under the influence of the electromagnetic field and a dipole moment is produced. As the electromagnetic waves in the atmosphere propagate just slightly slower than in a vacuum, refractive index $(n)$ is more conveniently expressed by refractivity, $\mathrm{N}$ :

$$
N=10^{6}(n-1)
$$

At every point of atmosphere the refractive index of a parcel of air can be expressed as a function of atmospheric pressure, temperature and humidity (Bean, B.R. and E.J. Dutton, 1996). However Davis et al. proposed an alternative separation of the refractivity components (Davis et al., 1985); the first and second terms of below equation are called the hydrostatic and nonhydrostatic components of the refractivity respectively.

$$
N=K_{1} R_{d} \rho+\left[K_{2}^{\prime}\left(\frac{e}{T}\right)+K_{3}\left(\frac{e}{T^{2}}\right)\right] Z_{w}^{-1}
$$

Where $K_{1}, K_{2}^{\prime}$ and $K_{3}$ are refractivity constants. $\rho$ is the total density, $e$ is water vapor pressure and $T$ is the temperature. $Z_{w}$ is the compressibility factors to regarding the non-ideal gas behaviors for water vapor (Owens, J.C., 1967). Various researchers have determined value for refractivity constants. Rüeger provided a very comprehensive review and based on past insights recommends: $K_{1}=77.6890\left(\mathrm{~K} \mathrm{hPa}^{-1}\right), K_{2}=71.2952\left(\mathrm{~K} \mathrm{hPa}^{-1}\right), K_{3}=375463\left(\mathrm{~K}^{2} \mathrm{hPa}{ }^{-1}\right)$ and $K_{2}^{\prime}=$ 22.9741( $\left.\mathrm{K} \mathrm{hPa}^{-1}\right)$ to be "best average coefficients" (Rüeger J.M, 2002).

The total delay is defined as the difference between the electromagnetic path length and the geometric length (path of the ray in vacuum); after some efforts we obtain:

$$
d_{n a}=\int_{\text {ray }}(n-1) d s+\left[\int_{\text {ray }} d s-\int_{\text {vac }} d s\right]
$$

The first term is excess path delay due to the delay experienced by signal and second term is the geometric delay due to the bending of the ray. The geometric delay is zero for zenith delay. As discussed before, the refractivity can be divided to hydrostatic and non-hydrostatic component. Consequently, the zenith delay can also be split into two components, called the zenith hydrostatic delay $\left(d_{h}^{z}\right)$ and zenith non-hydrostatic delay $\left(d_{n h}^{z}\right)$.

or

$$
d_{n a}^{z}=10^{-6} \int_{r_{s}}^{r_{a}} N_{h} d z+10^{-6} \int_{r_{s}}^{r_{a}} N_{n h} d z
$$

$$
d_{n a}^{Z}=d_{h}^{Z}+d_{n h}^{Z}
$$

If a flat earth and a constant refractivity are assumed, the bending will also be zero. In this case, the neutral-atmosphere propagation delay at any elevation angle $(\varepsilon)$ can be computed by:

$$
d_{n a}=d_{n a}^{Z} \csc (\varepsilon)
$$


The assumption clearly is not accurate; however the neutral propagation delay can be related to zenith delay by a specific function called mapping function. Therefore, in general we have:

$$
d_{n a}=d_{h}^{Z} \cdot m_{h}(\varepsilon)+d_{n h}^{Z} \cdot m_{n h}(\varepsilon)
$$

Where $m_{h}(\varepsilon)$ is a hydrostatic mapping function and $m_{n h}(\varepsilon)$ is a non-hydrostatic mapping function. Numerous mapping functions have been developed. As a general rule, these mapping functions assume symmetry of the neutral atmosphere. It means the models do not encompass azimuth dependence in their parameterization. Five recent mapping functions that are used for propagation delay will be outlined in details.

\section{MAPPING FUNCTIONS:}

Marini continued fractional form is the main functional formulation for development of most mapping functions (Marini, 1972):

$$
m(\varepsilon)=\frac{1+\frac{a}{1+\frac{b}{1+\frac{c}{a}}}}{\sin \varepsilon+\frac{a}{\sin \varepsilon+\frac{b}{\sin \varepsilon+\frac{c}{\cdots}}}}
$$

Researchers used the expression from eq. 8 to calibrate coefficients by means of a least squares fit to several ray-traced calculated between low elevation angle (e.g. $3.3^{\circ}$ ) to zenith. In the following, recent mapping functions are reviewed:

- Niell mapping function (NMF):

Niell proposed both a hydrostatic and non-hydrostatic mapping function based on the Marini functional formulation, truncated at the third term (Niell, 1996). The coefficients were calibrated by a ray-tracing campaign in nine elevation angles from $3^{\circ}$ to $90^{\circ}$, performed through temperature and humidity profiles at known pressure levels obtained from the U.S. Standard atmosphere:

$$
m(\varepsilon)=\frac{1+\frac{a}{1+\frac{b}{1+c}}}{\sin \varepsilon+\frac{a}{\sin \varepsilon+\frac{b}{\sin \varepsilon+c}}}+H_{S} \times 10^{-3}\left[\frac{1}{\sin \varepsilon} \frac{1+\frac{a_{h t}}{1+\frac{b_{h t}}{1+c_{h t}}}}{\sin \varepsilon+\frac{a_{h t}}{\sin \varepsilon+\frac{b_{h t}}{\sin \varepsilon+c_{h t}}}}\right]
$$

The first term describes the elevation dependence of the hydrostatic and non-hydrostatic mapping function while the second term is a height correction for regarding the increasing thickness of the atmosphere in respect to the radius of the curvature of the Earth. The correction is merely applied for hydrostatic mapping function. The temporal variability of coefficients of hydrostatic mapping function was modeled by a sinusoid with a period of 365.25 days:

$$
\begin{aligned}
& a_{h}(\varphi, D o Y)=a_{h_{\text {avg }}}(\varphi)-a_{h_{a m p}}(\varphi) \cos \left(2 \pi \frac{D o Y-28}{365.25}\right) \\
& b_{h}(\varphi, D o Y)=b_{h_{\text {avg }}}(\varphi)-b_{h_{a m p}}(\varphi) \cos \left(2 \pi \frac{D o Y-28}{365.25}\right) \\
& c_{h}(\varphi, D o Y)=c_{h_{\text {avg }}}(\varphi)-c_{h_{a m p}}(\varphi) \cos \left(2 \pi \frac{D o Y-28}{365.25}\right)
\end{aligned}
$$

Where DoY is the day of the year and $\varphi$ is latitude. The mean value, avg, amplitude, amp and height correction coefficients can be obtained for specific latitude from table 1 and 2. 
For non-tabulated latitude, the coefficients can be obtained by linear interpolation. For seasonal differences of southern hemisphere a phase of $\pi$ must be added to the input of $\cos$ in eq.10-12. Unlike the coefficients of hydrostatic mapping function, the non-hydrostatic mapping function depends merely on latitude and no obvious temporal relationship was found. The coefficients of non-hydrostatic mapping function are listed in table3.

Table 1. The coefficients of hydrostatic mapping function of NMF.

\begin{tabular}{|c|c|c|c|c|c|}
\cline { 2 - 6 } \multicolumn{1}{c|}{} & \multicolumn{5}{c|}{ Latitude } \\
\cline { 2 - 6 } \multicolumn{1}{c|}{$15^{\circ}$} & $30^{\circ}$ & $45^{\circ}$ & $60^{\circ}$ & $75^{\circ}$ \\
\hline$a_{h_{\text {avg }}}$ & $1.2769934 \mathrm{e}^{-3}$ & $1.2683230 \mathrm{e}^{-3}$ & $1.2465397 \mathrm{e}^{-3}$ & $1.2196049 \mathrm{e}^{-3}$ & $1.2045996 \mathrm{e}^{-3}$ \\
\hline$b_{h_{\text {avg }}}$ & $2.9153695 \mathrm{e}^{-3}$ & $2.9152299 \mathrm{e}^{-3}$ & $2.9288445 \mathrm{e}^{-3}$ & $2.9022565 \mathrm{e}^{-3}$ & $2.9024912 \mathrm{e}^{-3}$ \\
\hline$c_{\text {avg }}$ & $62.610505 \mathrm{e}^{-3}$ & $62.837393 \mathrm{e}^{-3}$ & $63.721774 \mathrm{e}^{-3}$ & $63.824265 \mathrm{e}^{-3}$ & $64.258455 \mathrm{e}^{-3}$ \\
\hline$a_{h_{\text {amp }}}$ & 0 & $1.2709626 \mathrm{e}^{-5}$ & $2.6523662 \mathrm{e}^{-5}$ & $3.4000452 \mathrm{e}^{-5}$ & $4.1202191 \mathrm{e}^{-5}$ \\
\hline$b_{h_{\text {amp }}}$ & 0 & $2.1414979 \mathrm{e}^{-5}$ & $3.0160779 \mathrm{e}^{-5}$ & $7.2562722 \mathrm{e}^{-5}$ & $11.723375 \mathrm{e}^{-5}$ \\
\hline${ }^{c} h_{\text {amp }}$ & 0 & $9.0128400 \mathrm{e}^{-5}$ & $4.3497037 \mathrm{e}^{-5}$ & $84.795348 \mathrm{e}^{-5}$ & $170.37206 \mathrm{e}^{-5}$ \\
\hline
\end{tabular}

Table 2. The coefficients of height correction.

\begin{tabular}{|l|l|}
\hline$a_{h t}$ & $2.53 \mathrm{e}-05$ \\
\hline$b_{h t}$ & $5.49 \mathrm{e}-03$ \\
\hline$c_{h t}$ & $1.14 \mathrm{e}-03$ \\
\hline
\end{tabular}

Table 3. The coefficients of non-hydrostatic mapping function of NMF.

\begin{tabular}{|c|c|c|c|c|c|}
\cline { 2 - 6 } \multicolumn{1}{c|}{} & \multicolumn{5}{c|}{ Latitude } \\
\cline { 2 - 6 } \multicolumn{1}{c|}{} & $15^{\circ}$ & $30^{\circ}$ & $45^{\circ}$ & $60^{\circ}$ & $75^{\circ}$ \\
\hline$a_{n}$ & $5.8021897 e^{-4}$ & $5.6794847 e^{-4}$ & $5.8118019 e^{-4}$ & $5.9727542 e^{-4}$ & $6.1641693 e^{-4}$ \\
\hline$b_{n}$ & $1.4275268 e^{-3}$ & $1.5138625 e^{-3}$ & $1.4572752 e^{-3}$ & $1.5007428 e^{-3}$ & $1.7599082 e^{-3}$ \\
\hline$c_{n}$ & $4.3472961 e^{-2}$ & $4.6729510 e^{-2}$ & $4.3908931 e^{-2}$ & $4.4626982 e^{-2}$ & $5.4736038 e^{-2}$ \\
\hline
\end{tabular}

- Vienna Mapping Functions (VMF1)

Vienna Mapping Functions (VMF1) proposed by (Boehm, J. et al., 2006b) is based on raytracing through the European Center of Mid-range Weather Forecasting (ECMWF). The functional formulation opted for this mapping function again was eq.8, truncated at three coefficients. The $b$ and $c$ coefficients for hydrostatic mapping function were determined by raytracing through ECMWF 40 years data in 9 diverse elevation angles. The temporal variation of the hydrostatic $c$ coefficient is modeled by a modified sinusoidal model given by:

$$
c=c_{o}+\left[\left(\cos \left(\frac{D o Y-28}{365} 2 \pi+\psi\right)+1\right) \times \frac{c_{11}}{2}+c_{10}\right] \times(1-\cos \varphi)
$$

The $\psi$ specifies the northern $(0)$ or southern $(\pi)$ hemisphere. The $b, c_{0}, c_{10}$ and $c_{11}$ are listed in table4: 
Table 4. The coefficients for hydrostatic VMF1

\begin{tabular}{|c|c|c|c|c|}
\hline Hemisphere & $C_{0}$ & $C_{10}$ & $C_{11}$ & \\
\hline Northern & 0.062 & 0.001 & 0.005 & 0 \\
\hline Southern & 0.062 & 0.002 & 0.007 & $\pi$ \\
\hline
\end{tabular}

The non-hydrostatic coefficients are fixed to NMF values at $45^{\circ}$ latitude. (i.e. $b_{\text {nh }}=0.00146$ and $\mathrm{c}_{\mathrm{nh}}=0.04391$ ). Unlike the NMF, VMF1 requires real-time data for measuring the $a$ coefficient for both hydrostatic and non-hydrostatic mapping function. It is measured by ray-tracing at an outgoing elevation angle of $3^{\circ}$ and then inverting the Marini expression. The $a$ coefficient for both hydrostatic and non-hydrostatic mapping function has been gridded on a $2.0 \times 2.5$ spatial resolution available from 1994 to present at (Website1).

- Global Mapping Functions (GMF):

Because the real-time data is needed to be used in VMF1, Boehm et al. calculated the $a$ coefficient for GMF using 15x15 global grids of monthly mean profiles of pressure, temperature and humidity from 3 years of ECMWF data and provided as a spherical harmonic expansion of degree and order 9 (Boehm, J. et al., 2006a):

where:

$$
a=a_{o}+A \cos \left(\frac{D o Y-28}{365} 2 \pi\right)
$$

$$
a_{o}=\sum_{n=0}^{9} \sum_{m=0}^{n} P_{m n}(\sin \varphi) \cdot\left[A_{m n} \cos (m \cdot \lambda)+B_{m n} \sin (m \cdot \lambda)\right]
$$

where A are the annual amplitudes, $P_{m n}(\sin \varphi)$ are the Legendre associated functions of $n$ degree and $m$ order, and $A_{m n}$ and $B_{m n}$ are the spherical harmonic coefficients. The $b$ and $c$ coefficients are the same as VMF1 parameters.

- Global Pressure and Temperature (GPT2)/GMF:

Regarding to eq.2, the hydrostatic component of refractivity completely depends on total density. It is straightforwardly can be obtained from the surface pressure (Saastamoinen, 1973). The GPT model was proposed to estimate temporal and spatial pressure and temperature variability (Boehm et al., 2007). Like the GMF, GPT is based on a spherical harmonic expansion (mean values and annual signal) of degree and order 9 derived from 3 years of ECMWF data. GPT2 is a new version of previous model. A refined horizontal resolution of $5^{\circ}$, an incorporating semiannual harmonic in order to consider very rainy periods or very dry periods regions, a considering semi-annual variation of temperature lapse rate and finally newer and longer mean profiles with greater pressure levels than GPT, are the main improvements of the new version (Lagler et al., 2013). The new model does not have merely surface measurements but $\mathrm{p}, \mathrm{T}$, specific humidity, Q, profiles and lapse rate; therefore the coefficients $a$ of the hydrostatic and non-hydrostatic mapping function were calculated for every profile using the VMF1-approach as given in (Boehm et al., 2006b). Consequently, by using the GPT2, the $a$ can easily be obtained and be used directly as input of to the $V M F 1 . f$ subordinate (without any need of download)

- University of New Brunswick-VMF1 (UNB-VMF1):

In availability of other NWMs, Marcelo C. Santos et al. provided the same orography, output file format, and grid definition as defined by the VMF1 service with different NWM (Marcelo C. Santos, 2012). NCEP's Re-Analysis I and the Canadian Meteorological Centre's Global Deterministic Prediction System (GDPS) were utilized with a different ray-tracing algorithm 
(Nievinski, 2009) to obtain $a$ coefficients, while $b$ and $c$ coefficients are the same parameterization of the VMF. Since two different NWM's have been used, the service provides two separated products: unbvmfG with 2.0x2.5 degree global grid NCEP Re-Analysis, 7 day latency, from 2001; unbvmfGcmc with 2.0x2.5 degree global grid CMC - GDPS, 1 day latency, from 2012 (Website2). In this work, we simply call the first product UNB-NCEP and second one, UNB-CMC, although not totally accepted, these terms may be more distinguishable.

As an instance, hydrostatic and non-hydrostatic NMF, VMF1, GMF, GPT2/GMF, UNB-NCEP and UNB-CMC on 1/1/2012 for $5^{\circ}$ elevation angle for the whole world have been determined:

Table 5. Hydrostatic and non-hydrostatic mapping function on $1 / 1 / 2012$ for $5^{\circ}$ elevation angle (part 1)

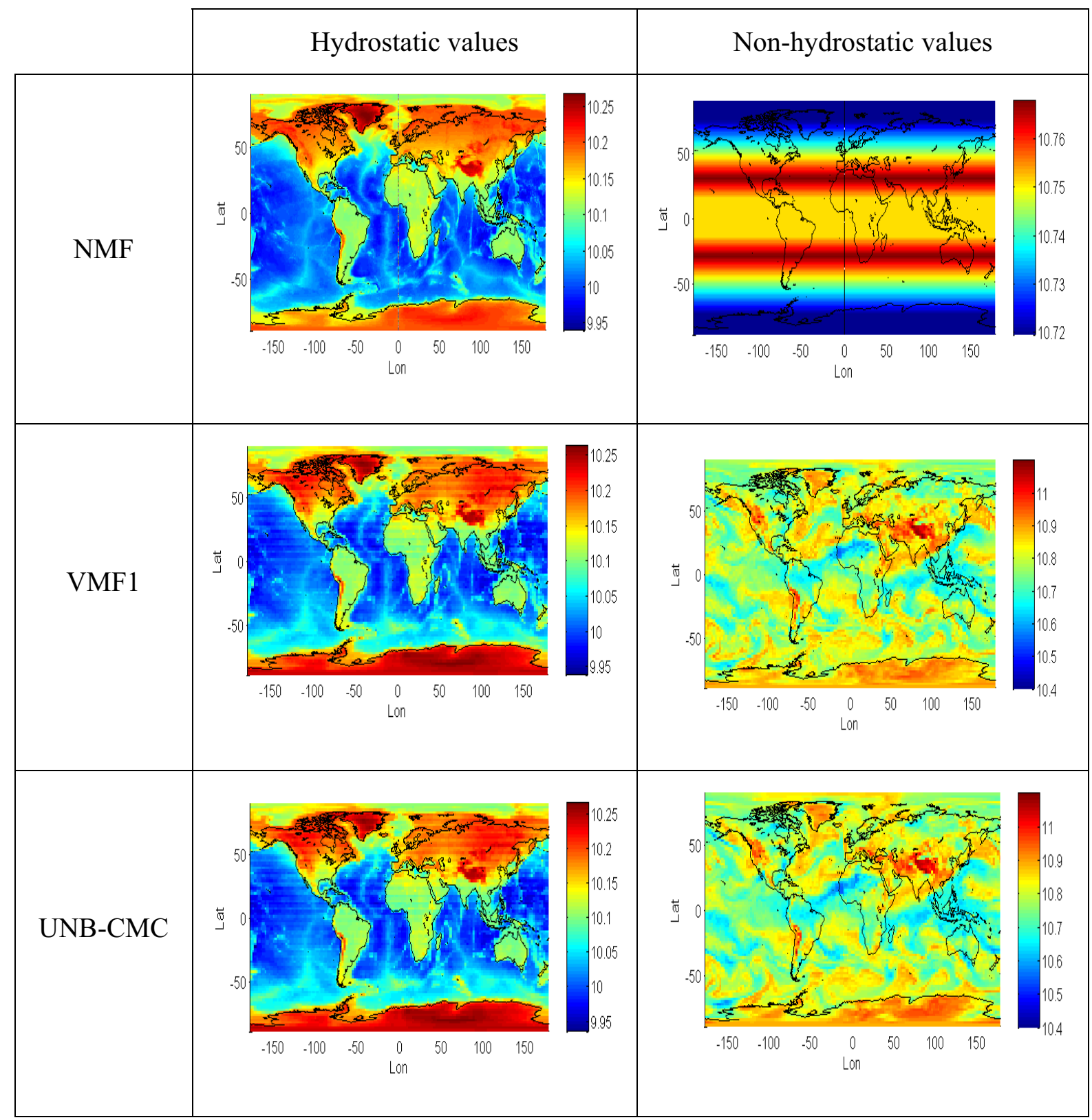


Table 5. Hydrostatic and non-hydrostatic mapping function on 1/1/2012 for $5^{\circ}$ elevation angle (part 2)

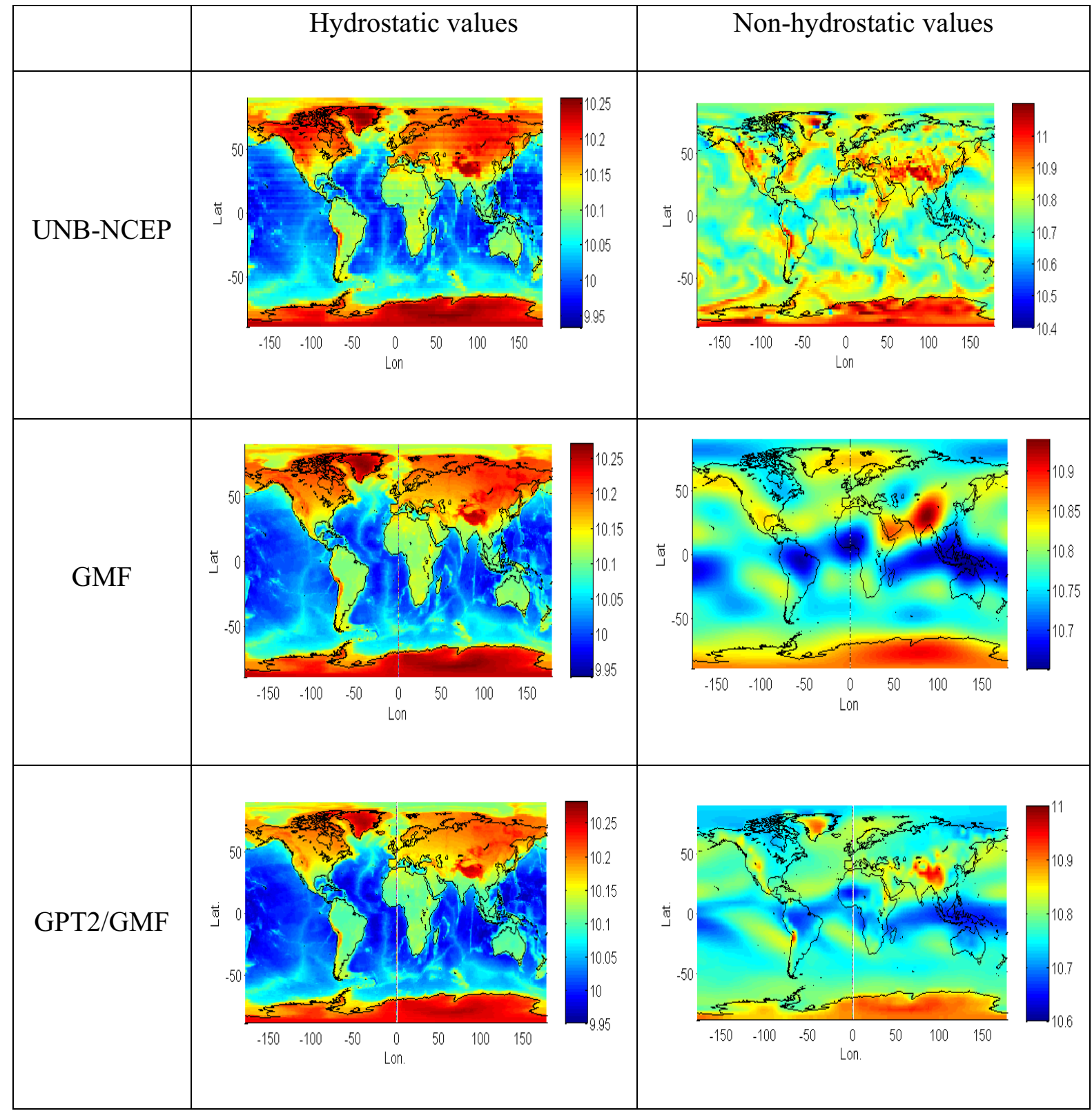

According to table 5, not surprisingly, the spatial pattern of hydrostatic component varies very smoothly and there is a hardly difference between mentioned hydrostatic mapping functions. On the other hand, offline mapping functions are able to express only a small fraction of the water vapor variability; particularly, NMF is incapable of modeling continuous changes of water vapor over the globe. Apparently, the online mapping functions do not suffer from water vapor fluctuations. 
The notion of spatio-temporal variations is based on the changes of a spatial variable (latitude) over time (month). As an instance, spatio-temporal variations of hydrostatic and non-hydrostatic NMF, VMF1, GMF, GPT2/GMF, UNB-NCEP and UNB-CMC for $5^{\circ}$ elevation angle in $180^{\circ} \mathrm{E}$ longitude have been determined:

Table 6. Spatio-temporal variations of hydrostatic and non-hydrostatic mapping function for $5^{\circ}$ elevation angle in $180^{\circ} \mathrm{E}$ longitude (part 1).

\begin{tabular}{|c|c|c|c|c|}
\hline & Hydrostatic values & & Non-hydrostatic values & \\
\hline NMF & 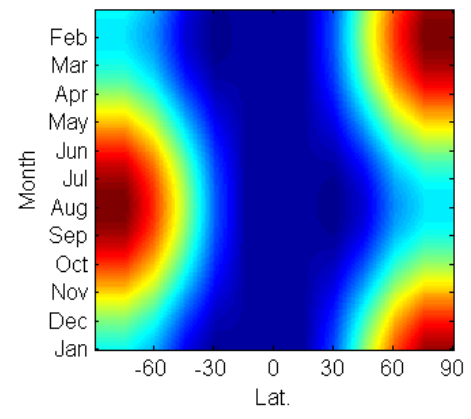 & $\begin{array}{r}10.18 \\
10.16 \\
-10.14 \\
10.12 \\
10.1\end{array}$ & 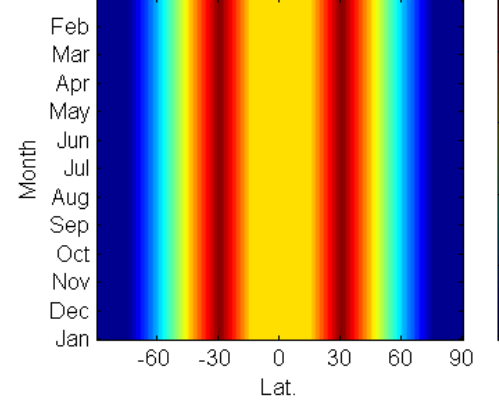 & $\begin{array}{l}10.76 \\
-10.75 \\
10.74 \\
10.73 \\
10.72\end{array}$ \\
\hline VMF1 & 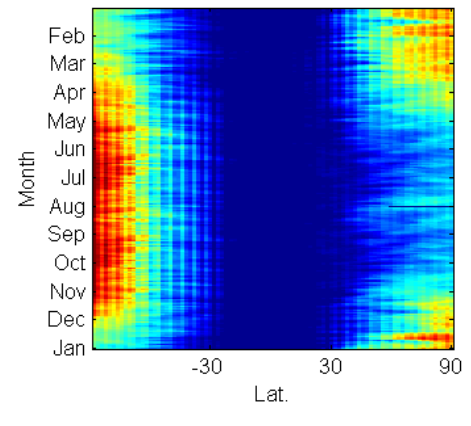 & 10.2 & 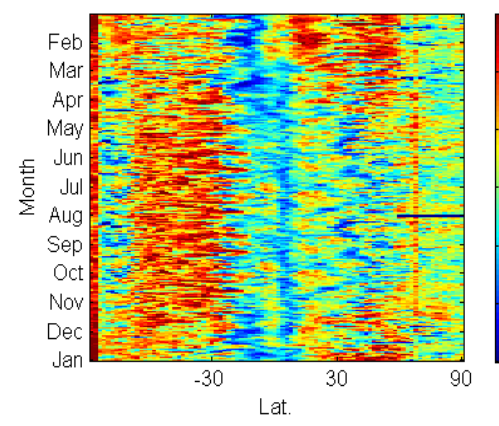 & $\begin{array}{l}10.85 \\
10.8 \\
10.75 \\
10.7 \\
10.65 \\
10.6\end{array}$ \\
\hline UNB-CMC & 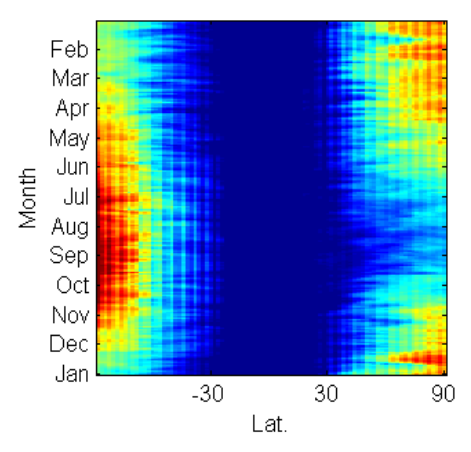 & $\begin{array}{l}10.2 \\
10.15\end{array}$ & 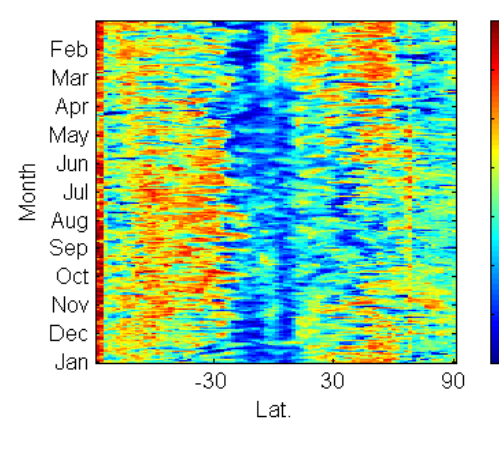 & \begin{tabular}{l|}
10.95 \\
10.9 \\
10.85 \\
10.8 \\
10.75 \\
10.7 \\
10.65 \\
10.6
\end{tabular} \\
\hline
\end{tabular}


Table 6. Spatio-temporal variations of hydrostatic and non-hydrostatic mapping function on $1 / 1 / 2012$ for $5^{\circ}$ elevation angle in $180^{\circ} \mathrm{E}$ longitude (part 2).

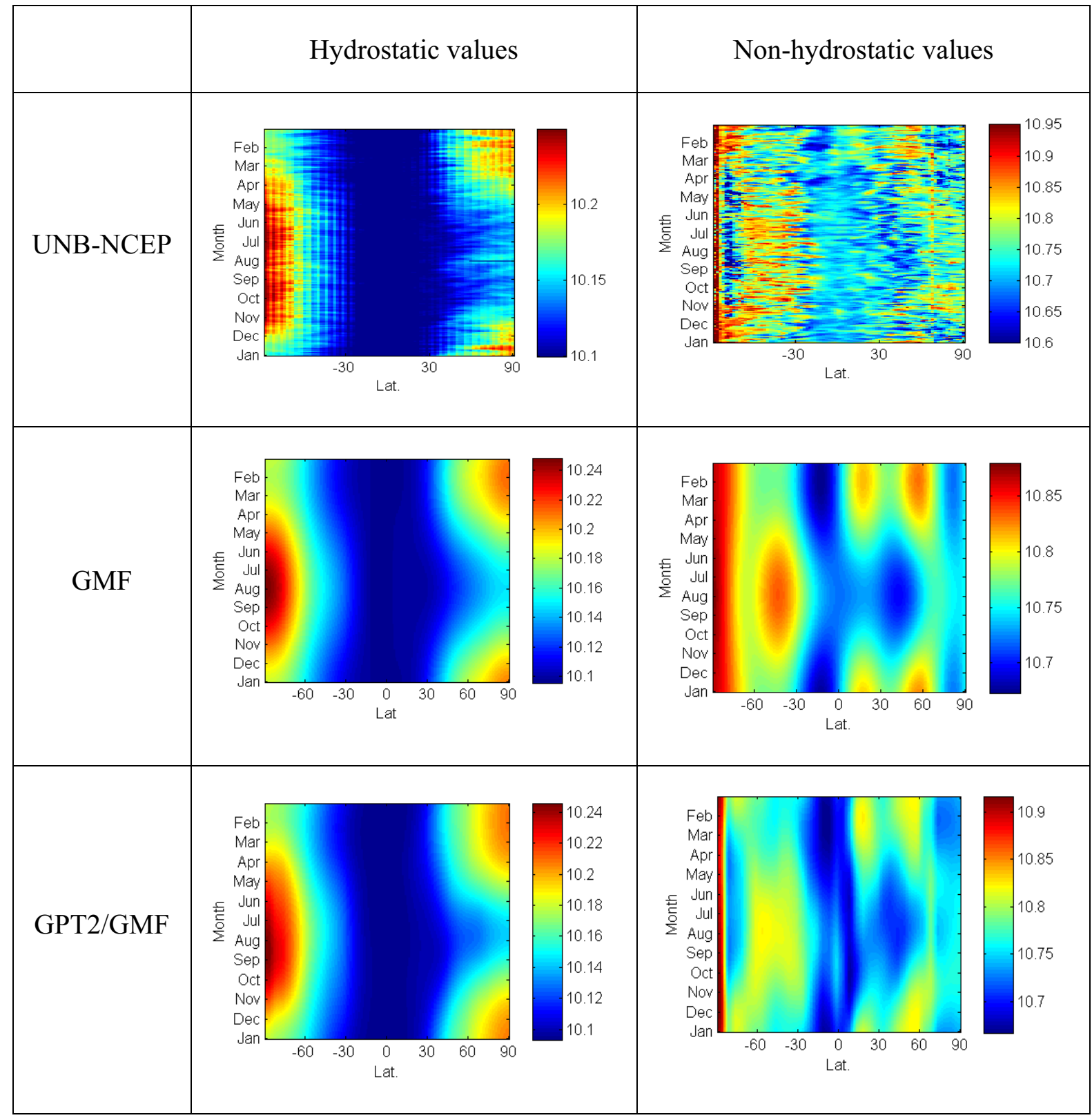

The shown table reveals one important characteristic of hydrostatic changes that makes it interesting for interpretation of mapping functions: large amplitudes changes in surface pressure occur substantially over Antarctica. It was proposed in (Parish and Bromwich, 1997) that such large pressure changes reflect variations in the mean mass transports to and from the antarctic 
continent. Such transports are accomplished by a meridional circulation between the continent and subpolar latitudes; the antarctic katabatic wind regime is thought to be a critical component of the lower branch of the meridional circulation (Parish and Bromwich, 1997). The seasonal variations of hydrostatic components experienced by the northern and southern hemisphere are in opposite direction. Another point to consider is the ability of online mapping function to quantify subtle variations of water vapor.

To summarize the main differences between the mentioned mapping functions, a review is provided in table 7:

Table 7. Summary of the characteristics of the state-of-the-art mapping functions evaluated in this paper.

\begin{tabular}{|c|c|c|c|c|c|c|}
\hline & NMF & VMF1 & GMF & GPT2/VMF1 & UNB-NCEP & UNB-CMC \\
\hline $\begin{array}{l}\text { Atmospheric } \\
\text { source }\end{array}$ & $\begin{array}{l}\text { U.S Standard } \\
\text { Atmosphere }\end{array}$ & ECMWF & $\begin{array}{c}\text { Mean } \\
\text { ECMWF, (23 } \\
\text { pressure } \\
\text { levels): } 1999- \\
2002)\end{array}$ & $\begin{array}{c}\text { Mean } \\
\text { ECMWF, (37 } \\
\text { pressure } \\
\text { levels): } 2001- \\
2010\end{array}$ & $\begin{array}{c}\text { NCEP's Re- } \\
\text { Analysis I }\end{array}$ & $\begin{array}{c}\text { CMC- } \\
\text { Global } \\
\text { Deterministic } \\
\text { Prediction } \\
\text { System } \\
\text { (GDPS) }\end{array}$ \\
\hline Representation & $\begin{array}{c}\text { Coefficients in } \\
\text { latitudinal } 15^{\circ} \\
\text { resolution }\end{array}$ & $\begin{array}{l}2.0^{\circ} \times 2.5^{\circ} \\
\text { spatial } \\
\text { resolution } \\
\text { (on-line) }\end{array}$ & $\begin{array}{l}\text { Spherical } \\
\text { harmonics up } \\
\text { to degree and } \\
\text { order } 9 \text { at } \\
\text { mean sea level } \\
\text { (horizontal } \\
\text { resolution of } \\
\text { about } 20^{\circ} \text { ) }\end{array}$ & $\begin{array}{c}5^{\circ} \text { grid at } \\
\text { mean } \\
\text { ETOPO5- } \\
\text { based heights }\end{array}$ & $\begin{array}{l}2.0^{\circ} \times 2.5^{\circ} \\
\text { spatial } \\
\text { resolution } \\
\text { (on-line) }\end{array}$ & $\begin{array}{l}2.0^{\circ} \times 2.5^{\circ} \\
\text { spatial } \\
\text { resolution } \\
\text { (on-line) }\end{array}$ \\
\hline
\end{tabular}

\section{SUMMARY}

In this work, a variety of the most recent mapping functions have been discussed. Three of them (NMF, GMF, GPT2/GMF) do not need any specific data as an input, however they have some shortcomings. They roughly modeled the spatial and temporal variation of water vapor and the temporal variation of hydrostatic component particularly in Polar Regions. As another weakness, their spatial resolution is too low. In general, a period of the studies of neutral atmosphere mapping functions, based on generic models and on-line measurements, may be considered to be completed. However, there are a number of challenging questions. Some of wish, if answered, 
could enhance our knowledge of how the mapping functions affect geodetic-positioning. One of the primary issues is related to the following question: How accurate are the mapping functions to model the turbulent atmosphere? Accordingly, as a future work, mapping functions errors, specifically standard deviation of error in respect to latitude, will be evaluated carefully using some benchmarks provided by ray-tracing through radiosonde measurements.

\section{REFERENCES}

AGU, December 1995: Water vapor in the climate system special report: http://www.eso.org/gen-fac/pubs/astclim/espas/pwv/mockler.html

Bean, B.R. and E.J.Dutton, 1996: Radio Meteorology. National Burea of Standards Monograph 92, U.S Goverment Printing Office: Washington, D.C.

Boehm J, Heinkelmann R, Schuh H, 2007: Short note: a global model of pressure and temperature for geodetic applications. J Geodesy, Vol.81, No.10, pp. 679-683. doi:10.1007/s00190-007-0135-3

Boehm, J., A. E. Niell, P. Tregoning, and H. Schuh, 2006a: Global Mapping Function(GMF): A new empirical mapping function based on numerical weather model data. Geophysical Research Letters, Vol. 33, No. L07304, doi:10.1029/2005GL025546.

Boehm, J., B. Werl, and H. Schuh, 2006b: Troposphere mapping functions for GPS and very long baseline interferometry from European Centre for Medium-Range Weather Forecasts operational analysis data. Journal of Geophysical Research, Vol. 111, No.B02406.

Davis, J.L., T.A.Herring, I.I.Shapiro, A.E.E. Rogers and G.Elgered, 1985: Geodesy by radio interferometry: Effects of atmospheric modeling errors on estimated of baseline length. Radio science, Vol.20, No.6, pp. 1593-1607.

Emardson, T. R., G. Elgered, and J. M. Johansson, 1998: Three months of continuous monitoring of atmospheric water vapor with a network of Global Positioning System receivers. J. Geophys. Res., Vol.103, pp. 1807-1820

K. Lagler, M. Schindelegger,J. Böhm, H. Krásná,and T.Nilsson, 2013: GPT2: Empirical slant delay model for radio space geodetic techniques, Geophysical Research Letters, Vol. 40, pp. 1069-1073, doi:10.1002/grl.50288.

Marcelo C. Santos, Matthew McAdam, and Johannes Boehm, 2012: Implementation Status of the UNB-VMF1, EGU General Assembly 2012 Vienna, Austria, pp. 22- 27.

Marini, J. W. 1972: Correction of satellite tracking data for an arbitrary tropospheric profile, Radio Science, Vol. 7, No. 2, pp. 223-231, doi:10.1029/RS007i002p00223.

Mendes, V.B. 1999: Modeling The Neutral-Atmosphere Propagation Delay in Radiometric Space Techniques. Ph.D. dissertation, Department of Geodesy and Geomatics Engineering Technical Report No. 199, University of New Brunswick, Canada.

Niell, A. E., 1996: Global mapping functions for the atmosphere delay at radio wavelengths. Journal of Geophysical Research, Vol. 101, No. B2, pp. 3227-3246.

Niell, A. E., A. J. Coster, F. S. Solheim, V. B. Mendes, P. C. Toor, R. B. Langley, and C. A. Upham, 2001: Comparison of measurements of atmospheric wet delay by radiosonde, water vapor radiometer, GPS, and VLBI. J. Atmos. Oceanic Technol., Vol.18, pp. 830-850 
Nievinski, F. G., 2009: Ray-tracing Options to Mitigate the Neutral Atmosphere Delay in GPS. M.Sc.E. thesis, University of New Brunswick, Dept. of Geodesy and Geomatics Engineering, Fredericton, N.B., Canada, May, 232 pp., Technical Report 262,

Owens, J.C., 1967: Optical refractive index of air: Dependence on pressure, temperature and composition. Applied Optics, Vol.6, No.1,pp. 51-59

Parish, T. R., and D. H. Bromwich, 1997: On the forcing of seasonal changes in surface pressure over Antarctica. Journal of Geophysical Research, 102(D12), pp. 13785-13792, doi:10.1029/96JD02959.

Rüeger, J.M, 2002: Refractive index formula for radio waves. FiG XXII International Congres, International Federation of Surveyors(FIG), Washington,D.C, April 19-26.

Saastamoinen, J., 1973: Contributions to the theory of atmospheric refraction. In three parts. Bulletin Geodesique, No.105, pp. 279-298; No.106, pp. 383-397; No.107, pp. 13-34.

Website1: http://ggosatm.hg.tuwien.ac.at

Website2: http://unb-vmf1.gge.unb.ca/pub/

Received: 2013-07-26,

Reviewed: 2013-09-30,

Accepted: 2013-11-20. 